\title{
The Establishment of a Cryo-EM Facility and Workflows to Support Pipeline Projects at Genentech
}

\author{
Alberto Estevez ${ }^{1}$, Chris Arthur ${ }^{1}$, Alexis Rohou ${ }^{1}$, and Claudio Ciferri ${ }^{1}$ \\ 1. Department of Structural Biology, South San Francisco, California, USA.
}

A new era of cryo-EM has begun to take shape as Big Pharma, consortia, and others complete their high investment decision to establish cryo-EM workflows and update the toolbox of techniques used to investigate proteins involved in human disease. This has come about due to the highly publicized "resolution revolution" which has propelled this technology into the forefront as a way to address questions regarding protein structure and protein-protein interactions. With these systems in place, leadership and departments tasked with elucidating structural data look to these facilities to establish efficient workflows designed to guide product development and basic research. The ability to visualize small molecules bound to target proteins is incredibly valuable to discovering and improving new drugs. Additionally, the ability to observe higher order structure at low resolution can quickly inform large molecule programs through techniques like epitope mapping [1] and observing conformational heterogeneity.

As facilities come on line there tends to be two models of cryo-EM workflows. One relies on outsourcing of the high-resolution data collection to a core facility located far from the investigator. In this model, screening of cryo-grids can be done in-house on a lower energy, cheaper, tool. Only the best grids are sent for imaging, thereby being efficient with the time purchased on an external microscope. The high demand for microscope time at most facilities, as well as the effort to schedule and send a sample, can make the turnaround time at this step exceedingly long. This can be particularly painful for projects that require an iterative process.

The second model, which we have adopted at Genentech, relies on building an EM facility in-house in order to have tighter control of the workflow from DNA to structure. This model complements our robust pipeline infrastructure and expertise in generating proteins, determining structures, and describing how proteins interact with various partners. Beginning with in-house expertise on plasmid design and curation, researchers can quickly design a plan to address questions at the DNA level. Constructs containing various tags or modifications can then be triaged using the expertise of expression and purification groups which perform high throughput analysis on multiple constructs in multiple expression systems simultaneously. This is a powerful tool in guiding large scale protein production. Labs tasked with providing the project with important data can then attempt to provide samples for imaging in EM, crystallization trials, or analysis by various other methods. They also use techniques such as fluorescent size exclusion chromatography and dynamic light scattering as well as biochemical methods to better characterize samples.

The first phase of our facility is designed to house two microscopes with a long-term vision to accommodate up to five microscopes. The primary tool for the front end of the imaging workflow is the Talos F200C (Talos) (ThermoFisher Scientific, Waltham, MA). The Talos handles all negative stain activities and serves for screening cryo samples before moving onto our higher energy tool. For high resolution data collection, we use a Titan Krios with a BioQuantum energy filter and a K2 camera (Gatan, 
Pleasanton, CA). Both microscopes are outfitted with automated data acquisition software (EPU (ThermoFisher) and Serial EM (Boulder laboratory for 3D electron microscopy) for data collection.

Access to strong bioinformatics support is also designed into our workflow. With large computer clusters we can implement image processing software such as cisTEM (cistem.org), to characterize the quality of data coming from both microscopes in a short amount of time. The increase in data and activity around these workflows has also driven the need for software to capture these activities. To this end we have developed software to track and record details of samples handled within our EM workflows (A. Rohou et al. unpublished).

Our facility is designed to grow and incorporate new technologies as they become available. Towards this end we will evaluate the use of the volta phase plate (ThermoFisher) on our Titan Krios in the upcoming year. We will also explore the use of micro electron diffraction (microED) as a technique for structure determination in our department thus adding a new function to our Talos microscope.

At Genentech we are fortunate to have tremendous amounts of expertise in the same location working toward the same goal. Our approach is aimed towards lowering turnaround time and speeding up project progression.

\section{References:}

[1] Ciferri C. et al, M\&M 2016.

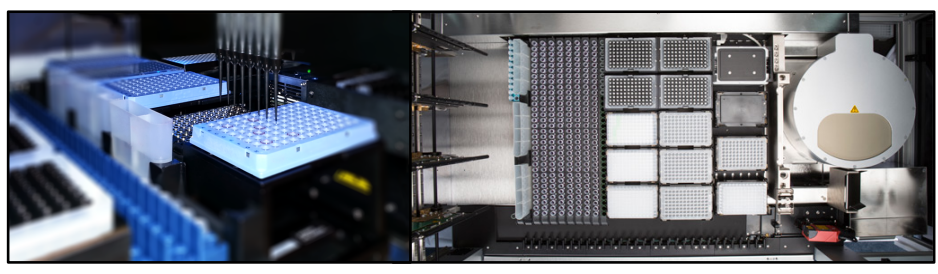

Figure 1. Construct generation, protein production and purification in a high throughput format. Protein purification from protein expression cultures purified on a Biomek FX (Beckman Coulter) or a Microlab Star (Hamiliton) liquid handler.

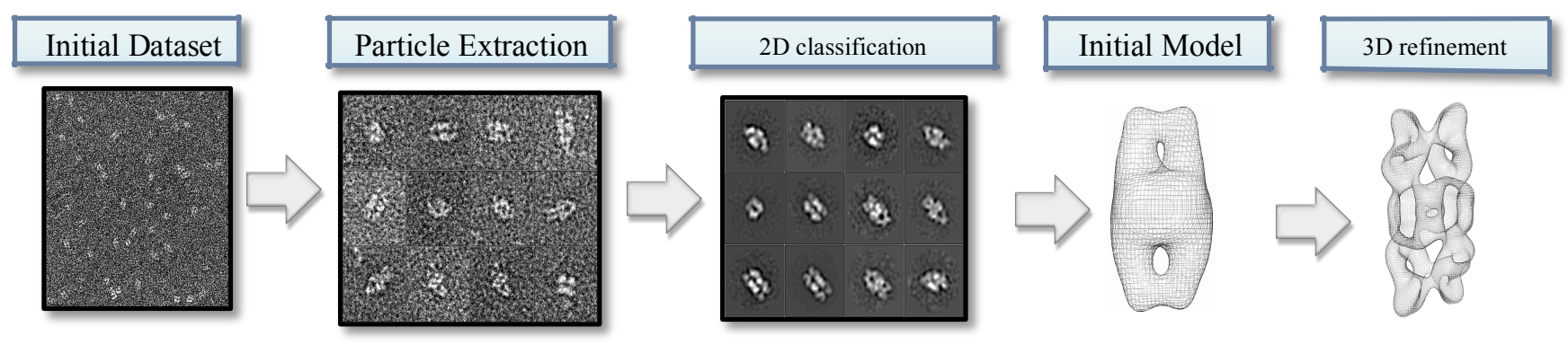

Figure 2. Canonical approach to 3D map generation in the cryo-EM and negative stain workflow. Data collected at each phase can aid n sample characterization. 